\title{
Caracterización de la lesión deportiva en atletas caucanos con proyección a Juegos Deportivos Nacionales
}

\author{
Characterization of the sports injury in athletes from the department of Cauca with projection to National Sports \\ Games
}

Andrés Felipe Villaquirán1*, Enmanuel Portilla-Dorado², Paola Vernaza-Pinzón³

1. Fisioterapeuta. Mg en Intervención Integral en el Deportista. Docente Departamento de Fisioterapia, Facultad de Ciencias de la Salud, Universidad del Cauca. Popayán, Colombia. e-mail: avillaquiran@unicauca.edu.co

2. Fisioterapeuta y Licenciado en Educación Física. Docente Departamento de Educación Física, Facultad de Educación, Universidad del Cauca. Popayán, Colombia. e-mail: efportilla@unicauca.edu.co

3. Fisioterapeuta. Esp. en Epidemiología General. Docente Titular Departamento de Fisioterapia, Facultad de Ciencias de la Salud, Universidad del Cauca. Popayán, Colombia. e-mail: pvernaza@unicauca.edu.co orcid.org/0000-0002-8918-4736

\begin{abstract}
Resumen
Objetivo: Caracterizar las lesiones deportivas en atletas caucanos, que se preparan para su participación en los Juegos Deportivos Nacionales 2015. Materiales y métodos: Estudio descriptivo de corte transversal realizado con los deportistas caucanos con proyección a Juegos Deportivos Nacionales, atendidos en el servicio de Fisioterapia de la Unidad Biomédica de Indeportes Cauca, durante el período comprendido entre diciembre de 2013 y julio de 2015; la información fue colectada a través de la ficha de características socio demográficas y clínicas de la Unidad Biomédica de Indeportes, Cauca; las variables estudiadas fueron: edad, sexo, estrato, mes de atención, deporte practicado, zona del cuerpo lesionada, tipo de lesión y diagnóstico médico de remisión. Resultados: El deporte que más lesiones presentó fue bádminton con el $100 \%$ de lesionados, seguido de judo con el 13,9\%, fútbol de salón con el 12,1\% y voleibol con el 8,7\%; las tendinopatías y las lesiones ligamentosas ocuparon el primer lugar de incidencia con el 50,6\% de las lesiones deportivas, la zona más comprometida correspondió a los miembros inferiores. Conclusiones: El estudio documenta las lesiones por cada práctica deportiva con la finalidad de realizar un programa de prevención y seguimiento a las lesiones, el cual redundará en el rendimiento de las ligas y en los resultados de los deportistas caucanos.
\end{abstract}

Palabras clave: Atletas; deportes; traumatismos en atletas; epidemiología; Colombia. (Fuente: DeCS, Bireme).

\begin{abstract}
Objective: To characterize the sports injuries in athletes from the department of Cauca, that are getting prepared for their participation in the National Sport Games in 2015. Materials and methods: A cross-sectional descriptive study was carried out with the athletes from Cauca with projection to National Sport games, who were attended in the physiotherapy service of the Biomedicine Unit of Indeportes Cauca during December 2013 and July 2015. The information was collected through the socio-demographic and clinical characteristics file of the Biomedicine Unit of Indeportes Cauca. The variables studied were: age, sex, stratum, month of attention, practiced sport, injured area of
\end{abstract}


the body, type of injury and medical diagnosis of remission. Results: The sport that presented more lesions was badminton with $100 \%$ of injured people, followed by Judo with $13.9 \%$, indoor soccer with $12.1 \%$ and volleyball with 8.7\%. The tendinopathies and ligament injuries occupied the first place of incidence with $50.6 \%$ of sports injuries, the most affected area was the lower limbs. Conclusions: The study documents the lesions each sport reports with the purpose of performing a program of prevention and follow-up to the injury, which will result in the performance of the leagues and the results of the sportsmen from Cauca.

Keywords: Athletes; sports; athletic injuries; epidemiology; Colombia. (Source: DeCS, Bireme).

\section{Introducción}

La actividad deportiva se asocia con un determinado riesgo de lesión, el incremento en la energía musculo-esquelética en los deportes de alto rendimiento tiene mayor probabilidad para que se presenten lesiones agudas y crónicas. Las lesiones deportivas han sido definidas como el hecho que ocurre durante una sesión de entrenamiento, programa o partido causando ausencia para la próxima sesión de entrenamiento ${ }^{1}$; Adamus y Nerin reportan que el Comité de Investigación del American Orthopaedic Society for Sports Medicine, define la lesión como el daño, percance o contratiempo que ocurra durante la competición o los entrenamientos y que causa baja en la competición o en dos o más días de entrenamiento o que conlleven a un descenso en la carga de entrenamiento en dos o más sesiones consecutivas ${ }^{2}$.

La búsqueda constante de resultados deportivos en el ámbito del alto rendimiento ha generado el aumento de la incidencia de la lesión deportiva, por ejemplo, cada año se producen 40 millones de lesiones músculo esqueléticas en los EE.UU, de las cuales 4 millones aproximadamente están relacionadas con los deportes ${ }^{3}$. Se ha calculado que la tasa de lesiones deportivas en la población general es de 15,4 por 1.000 personas y que la frecuencia promedio de lesiones en los atletas es de 5,2\% ${ }^{4}$. De acuerdo con el tipo de lesión, el 75\% de lesiones deportivas ocurren en los tejidos blandos produciendo: esguinces, calambres, desgarros, contusiones y abrasiones y se estima que de un $30 \%$ a $50 \%$ de las lesiones deportivas son causadas por uso excesivo de los tejidos blandos ${ }^{5}$.
Las lesiones deportivas son multicausales: las intrínsecas, tienen relación con las características personales, ya sean biológicas o psicológicas: morfología, condición física, predisposición al riesgo, condición individual de aprendizaje y experiencia que influyen en la técnica correcta y aptitudes hacia la práctica deportiva; y las extrínsecas se asocian al proceso de entrenamiento, equipamiento deportivo, la superficie, el nivel de competición y el clima, además de las exigencias del propio deporte ${ }^{6}$.

Sobre la clasificación, diferentes autores han sugerido la unificación de criterios en la metodología empleada para categorizar la lesión deportiva, las lesiones agudas ocurren cuando la carga tisular es lo suficientemente importante para ocasionar una deformación súbita e irreversible del tejido, estas son ocasionadas por caídas o golpes directos; mientras que las lesiones crónicas son a causa de una sobrecarga repetitiva, cuya acumulación a lo largo del tiempo excede el umbral de daño tisular, producto de un exceso de actividad, un aumento exagerado de la carga o un mal manejo de los tiempos de recuperación ${ }^{2}$.

Está claramente documentado en la literatura mundial, los efectos positivos de la actividad física en la salud, pero la actividad deportiva como actividad física especializada, organizada $\mathrm{y}$ reglamentada se asocia con un determinado riesgo de lesión, lo cual conlleva a realizar investigaciones epidemiológicas como un avance hacia los programas de prevención de lesiones en atletas $^{7}$. La epidemiología de las lesiones deportivas se convierte en la herramienta clave y fundamental para el estudio de la distribución y las variables que intervienen en la aparición de lesiones en grupos de población, con el propósito de establecer medidas preventivas ${ }^{8}$, y la vigilancia 
de estas las lesiones deportivas tiene como objetivo reducir el riesgo a partir de la identificación de la magnitud de lesiones, a través de la comprensión de las causas y los mecanismos de producción.

En Colombia, se reportan pocos estudios sobre lesiones deportivas. Un estudio realizado en la Universidad del Tolima describe las lesiones más prevalentes en la práctica del taekwondo destacándose las contusiones, seguidas de los esguinces ${ }^{9}$; el estudio de Sánchez y Castro sobre lesiones deportivas en patinaje reporta que el $80,8 \%$ de los atletas han presentado lesiones y el $43 \%$ han sufrido al menos 1 ó 2 lesiones a lo largo de su vida deportiva, adicionalmente el $42,9 \%$ de los patinadores de la liga presentaron lesión osea ${ }^{10 .}$

Actualmente, en el departamento del Cauca no existe una sólida investigación epidemiológica en medicina del deporte, por tanto, conocedores de la importancia de la prevención de las lesiones deportivas, en atletas de alto rendimiento, el objetivo de este primer estudio fue caracterizar la lesión deportiva en atletas caucanos que se preparan para los Juegos Deportivos Nacionales, los resultados del mismo entregarán insumos para implementar programas de prevención por parte de los cuerpos técnicos, grupos biomédicos y los organismos administrativos de cada deporte, quienes son los encargados de la preparación de los atletas en la búsqueda de los diferentes objetivos, metas y logros a nivel nacional e internacional, además de entregar un programa de prevención de lesiones deportivas.

\section{Materiales y métodos}

Se realizó un estudio descriptivo de corte transversal con los deportistas del departamento del Cauca de diferentes disciplinas, que se preparaban para los Juegos Deportivos Nacionales 2015, quienes fueron remitidos para su proceso de rehabilitación en la Unidad Biomédica de Indeportes Cauca. El estudio fue realizado durante el periodo diciembre de 2013 y julio de 2015. La población estuvo conformada por 417 atletas caucanos y la muestra correspondió a 231 deportistas lesionados y remitidos a la Unidad Biomédica. Como criterio de inclusión se tuvo en cuenta que los participantes figuraran en el listado de deportistas con proyección a Juegos Deportivos Nacionales, que fueran reconocidos por sus ligas como pertenecientes a las selecciones Cauca de cada disciplina deportiva; fueron excluidas del estudio aquellas personas que no se encontraban presentes para el diligenciamiento de la ficha socio demográfica y clínica y sujetos que no aceptaron participar en el presente estudio.

En el estudio participó el 100\% de la población intervenida. Las variables que se analizaron fueron: género, edad, ocupación, estrato, mes de atención, deporte practicado, zona del cuerpo lesionada, tipo de lesión y diagnóstico médico; con respecto al tipo de lesión se tuvo en cuenta el mecanismo de lesión y los síntomas, la clasificación para las lesiones agudas fueron el resultado de un hecho traumático como: un choque, un golpe o una carga tisular lo suficientemente grande para ocasionar una deformación súbita del tejido, mientras que las lesiones por sobrecarga presentaron un inicio lento e insidioso, debido a un exceso de carga de entrenamiento, dicha información se obtuvo de la ficha clínica. Las variables fueron registradas en una ficha de seguimiento del comportamiento de la lesión (formato de vigilancia epidemiológicaUnidad Biomédica Indeportes-Cauca), el formato, fue diligenciado por los fisioterapeutas de la Unidad Biomédica, luego de aprobada la participación de cada deportista con la firma del consentimiento informado.

El presente estudio hizo parte del proceso de investigación, contemplado dentro del micro currículo de la asignatura Énfasis en Deporte del Programa de Fisioterapia de la Universidad del Cauca y aprobado como estrategia metodológica por el comité de Plan del programa de fisioterapia y el Comité de la Unidad Biomédica de Indeportes-Cauca con fecha de noviembre 13 de 2013. Además, fue refrendado por el convenio docencia-servicio de ambas instituciones, todos los procedimientos siguieron las consideraciones éticas de la nueva Declaración de Helsinki11; para mantener la confidencialidad de la información de 
los sujetos estudiados, se asignó un código a cada ficha, para los sujetos menores de edad, el fisioterapeuta responsable de la Unidad Biomédica entregaba y explicaba a los entrenadores y tutores del deportista, los objetivos y el propósito de la presente investigación para luego proceder a firmar el asentamiento informado para la autorización de inclusión en la investigación.

Para el procesamiento y análisis estadístico de la información, se construyó una base de datos en el programa estadístico SPSS versión 22. Por ser un estudio de tipo descriptivo Las variables continuas se expresaron con la media \pm desviación estándar, y las variables discretas se expresaron en frecuencias y proporciones, dada la clasificación de las variables de estudio no se realizaron correlaciones.

\section{Resultados}

La muestra estuvo conformada por 231 individuos lesionados correspondientes al 55,3\% del total de los atletas preseleccionados en las diferentes disciplinas deportivas del departamento del Cauca. La población estudiada entrenaba en promedio 2,7 horas al día, 1,2 sesiones por día, una frecuencia de 7,2 sesiones a la semana, una media de exposición de 389.111 horas de entrenamiento al año, con un promedio de aparición de lesión de 0,6 lesión/1.000 horas.

El 55,4\% (128/231) de los deportistas pertenecía al sexo masculino y el 44,6 \% (103/231) al sexo femenino. La media de edad de los sujetos estudiados fue de 19 años, con una desviación estándar de $\pm 5,3$ años. Respecto a la escolaridad la mayoría de la población se encontraba estudiando, lo cual coincide con la ocupación, donde solo el 16,5\% (38/231), se dedicaban exclusivamente al deporte; en cuanto al estrato socioeconómico el 85,9\% (200/231) de los sujetos pertenecía a los estratos 1,2 y 3 ; y solo el $1,3 \%(3 / 231)$ de los encuestados eran casados o tenían una unión marital de hecho (Tabla 1).
Tabla 1. Características de la población de deportistas caucanos con proyección a Juegos Deportivos Nacionales, diciembre 2013 - julio 2015

\begin{tabular}{llrr}
\hline Variable & & N & \% \\
\hline Género & Masculino & 128 & 55,4 \\
Edad (años) & Femenino & 103 & 44,6 \\
& Media & \multicolumn{2}{c}{19} \\
& Mediana & \multicolumn{2}{c}{18} \\
Escolaridad & Mínimo & \multicolumn{2}{c}{41} \\
& Máximo & 47 \\
& Bachiller & 115 & 49,8 \\
Estrato & Técnico & 14 & 6,1 \\
socioeconómico & Universitario & 100 & 43,3 \\
& Posgrado & 2 & 0,9 \\
& 1 & 34 & 14,7 \\
Ocupación & 2 & 67 & 29,0 \\
& 3 & 99 & 42,9 \\
& 4 & 24 & 10,4 \\
& 5 & 7 & 3 \\
& Deportista & 38 & 16,5 \\
& Estudiante & 172 & 74,5 \\
& trabajador & 6 & 2,6 \\
& independiente & & \\
& Empleado & 13 & 5,6 \\
& Desempleado & 2 & 0,9 \\
& Soltero & 228 & 98,7 \\
& Casado & 3 & 1,3 \\
\hline
\end{tabular}

El total de la población de deportistas que hacían parte de las ligas fue la siguiente: 20 atletismo, 11 bádminton, 30 baloncesto, $7 \mathrm{Bmx}, 4$ boxeo, 6 ciclismo, 4 downhill, 42 futbol, 40 futbol de salón, 20 futbol sala, 3 golf, 50 judo, 15 karate, 15 levantamiento de pesas, 7 lucha, 10 motocross, 12 patinaje, 7 squash, 30 subacuáticas, 10 taekwondo, 10 tejo, 10 tenis, 4 tiro y caza, 10 triatlón y 40 voleibol ( Tabla 2).

Con respecto a los deportistas lesionados los resultados epidemiológicos encontrados reportan que, el deporte que más lesiones presento fue Bádminton con el $100 \%$ de lesionados, Judo con el 13,8\% (32/231), seguido de futbol de salón con 12,1\% (28/231) y voleibol con el 8,7\% (20/231), los deportes en los que menos lesionados se encontraron fueron tejo, tenis, tiro y caza, triatlón, lucha y golf con menos del 1\% (Tabla 2). 
Tabla 2. Relación de deportistas y deportistas lesionados en las ligas deportivas Departamento del Cauca, con proyección a Juegos Deportivos Nacionales

\begin{tabular}{|c|c|c|c|c|}
\hline Deporte & $\begin{array}{c}\text { Número total } \\
\text { deportistas }\end{array}$ & $\%$ & $\begin{array}{c}\text { Numero deportistas } \\
\text { lesionados }\end{array}$ & $\%$ \\
\hline Atletismo & 20 & 4,7 & 11 & 4,7 \\
\hline Bádminton & 11 & 2,6 & 11 & 100 \\
\hline Baloncesto & 30 & 7,1 & 6 & 2,5 \\
\hline Bmx & 7 & 1,6 & 4 & 1,7 \\
\hline Boxeo & 4 & 0,9 & 3 & 1,2 \\
\hline Ciclismo & 6 & 1,4 & 8 & 3,4 \\
\hline Downhill & 4 & 0,9 & 7 & 3,0 \\
\hline Futbol & 42 & 10,0 & 17 & 7,3 \\
\hline Futbol salón & 40 & 9,5 & 28 & 12,1 \\
\hline Futbol sala & 20 & 4,7 & 6 & 2,5 \\
\hline Golf & 3 & 0,7 & 2 & 0,8 \\
\hline Judo & 50 & 11,9 & 32 & 13,8 \\
\hline Karate & 15 & 3,5 & 8 & 3,4 \\
\hline Pesas & 15 & 3,5 & 15 & 6,4 \\
\hline Lucha & 7 & 1,6 & 2 & 0,8 \\
\hline Motocross & 10 & 2,3 & 4 & 1,7 \\
\hline Patinaje & 12 & 2,8 & 11 & 4,7 \\
\hline Squash & 7 & 1,6 & 2 & 0,8 \\
\hline A. Subacuáticas & 30 & 7,1 & 15 & 6,4 \\
\hline Taekwondo & 10 & 2,3 & 10 & 4,3 \\
\hline Tejo & 10 & 2,3 & 1 & 0,4 \\
\hline Tenis & 10 & 2,3 & 1 & 0,4 \\
\hline Tiro y caza & 4 & 0,9 & 1 & 0,4 \\
\hline Triatlón & 10 & 2,3 & 2 & 0,8 \\
\hline Voleibol & 40 & 9,5 & 20 & 8,6 \\
\hline Total & 417 & 100 & 231 & 100 \\
\hline
\end{tabular}

En la tabla 3, se pueden apreciar las características de la práctica deportiva en la población de estudio, encontrando que los deportes de pelota y raqueta tienen la mayor incidencia de lesión, referida ésta a la práctica de bádminton. El periodo de mayor ingreso a la unidad biomédica fue durante el primer trimestre del año 2014 correspondiente a los meses de enero, febrero y marzo con un 32,4\% (75/231).

Tabla 3. Características de la práctica deportiva de deportistas caucanos lesionados con proyección a Juegos Deportivos Nacionales

\begin{tabular}{|c|c|c|c|}
\hline Variable & & $\mathbf{n}$ & $\%$ \\
\hline \multirow[t]{4}{*}{ Clasificación de la práctica deportiva } & Combate & 55 & 23,8 \\
\hline & Tiempo y marca & 77 & 33,3 \\
\hline & Pelotas y raqueta & 97 & 42,0 \\
\hline & Arte y precisión & 2 & 0,9 \\
\hline \multirow{7}{*}{ Fecha de Ingreso a la Unidad Biomédica } & Diciembre 2013 & 6 & 2,5 \\
\hline & I trimestre de 2014 & 75 & 32,4 \\
\hline & II trimestre de 2014 & 46 & 19,9 \\
\hline & III trimestre de 2014 & 28 & 12,1 \\
\hline & IV trimestre 2014 & 18 & 7,7 \\
\hline & I trimestre de 2015 & 19 & 8,2 \\
\hline & II trimestre de 2015 & 40 & 17,3 \\
\hline
\end{tabular}


Respecto a las lesiones más frecuentes en los deportistas, la tendinitis rotuliana tuvo una incidencia del 9,7\% (21/231) seguida del esguince de tobillo grado I con una incidencia del $8,7 \%(20 / 231)$. Fueron un total de 143 diagnósticos los emitidos por el médico de la unidad biomédica, lo cual requirió realizar una categorización del diagnóstico médico de acuerdo a la deficiencia estructural: tendinopatía, lesión muscular, lesión ligamentosa, lesión ósea y lesión articular. Las tendinopatias y las lesiones ligamentosas ocuparon el primer lugar de incidencia con el 50,6\% de las lesiones deportivas en la población estudio (Tabla 4).
En la tabla 4, se describen las características epidemiológicas de la lesión deportiva, reportando un 60,6\% (140/231) de lesión, debido a sobrecarga del tejido, referidas estas lesiones a un exceso de carga de entrenamiento deportivo. Al determinar la zona de la lesión deportiva, se pudo observar que la mayor incidencia de lesión se presentó en los miembros inferiores con el 47,6\% (110/231), siendo las estructuras más afectadas rodilla con un $26 \%$ (60/231), tobillo con un $16 \%(37 / 231)$ y muslo con un $12,6 \%(29 / 231)$.

Tabla 4. Características epidemiológicas de la lesión deportiva de deportistas caucanos con proyección a Juegos Deportivos Nacionales

\begin{tabular}{|c|c|c|c|}
\hline Variable & & $\mathbf{n}$ & $\%$ \\
\hline \multirow[t]{5}{*}{ Clasificación de la lesión } & Agudas & 86 & 37,2 \\
\hline & Sobrecarga & 140 & 60,6 \\
\hline & Sobrecarga y aguda & 2 & 0,9 \\
\hline & Sobrecarga y sobrecarga & 2 & 0,9 \\
\hline & Otra (enfermedad) & 1 & 0,4 \\
\hline \multirow{5}{*}{$\begin{array}{l}\text { Categorización de la Lesión } \\
\text { deportiva }\end{array}$} & Tendinopatía & 63 & 27,3 \\
\hline & Lesión muscular & 61 & 26,4 \\
\hline & Lesión ligamentosa & 71 & 30,7 \\
\hline & Lesión ósea & 16 & 6,9 \\
\hline & Lesión articular & 20 & 8,6 \\
\hline \multirow[t]{12}{*}{ Mes de presentación de lesión } & Enero & 19 & 8,2 \\
\hline & Febrero & 33 & 14,3 \\
\hline & Marzo & 43 & 18,6 \\
\hline & Abril & 25 & 10,8 \\
\hline & Mayo & 38 & 16,5 \\
\hline & Junio & 17 & 7,4 \\
\hline & Julio & 20 & 8,7 \\
\hline & Agosto & 4 & 1,7 \\
\hline & Septiembre & 8 & 3,5 \\
\hline & Octubre & 7 & 3,0 \\
\hline & Noviembre & 7 & 3,0 \\
\hline & Diciembre & 10 & 4,3 \\
\hline \multirow[t]{4}{*}{ Zona lesionada } & Miembros superiores & 97 & 42,0 \\
\hline & Tórax y tronco & 22 & 9,5 \\
\hline & Miembros inferiores & 110 & 47,6 \\
\hline & Tronco y miembros inferiores & 2 & 0,9 \\
\hline
\end{tabular}

La tabla 5, describe el análisis bivariado por tipo de lesión y género, encontrando que tanto en hombres como en mujeres se presentó mayor afección de tejido ligamentario. Respecto a la clasificación del deporte: en el de combate hubo mayor incidencia de tendinopatías, al igual que en el deporte de tiempo y marca, mientras que en el de pelota y raquetas predominó la lesión ligamentosa; respecto a la clasificación de la lesión, la lesión de tipo traumático provocó más lesión en el tejido ligamentario, la lesión producida por sobrecarga produjo mayor lesión de tipo muscular y en cuanto a la zona lesionada, los miembros superiores fueron los más 
susceptibles a sufrir tendinopatías, mientras que estructuras del tórax y el tronco presentaron lesiones musculares, y los miembros inferiores fueron afectados por lesiones ligamentosas.

Tabla 5. Análisis bivariado de la lesión deportiva de deportistas caucanos con proyección a Juegos Deportivos Nacionales

\begin{tabular}{|c|c|c|c|c|c|c|c|c|c|c|}
\hline & \multicolumn{2}{|c|}{ Tendinopatía } & \multicolumn{2}{|c|}{$\begin{array}{c}\text { Lesión } \\
\text { muscular }\end{array}$} & \multicolumn{2}{|c|}{$\begin{array}{c}\text { Lesión } \\
\text { ligamentaria }\end{array}$} & \multicolumn{2}{|c|}{ Lesión ósea } & \multicolumn{2}{|c|}{ Lesión articular } \\
\hline & $\mathbf{n}$ & $\%$ & $\mathbf{n}$ & $\%$ & n & $\%$ & $\mathbf{n}$ & $\%$ & $\mathbf{n}$ & $\%$ \\
\hline \multicolumn{11}{|c|}{ Género } \\
\hline Femenino & 30 & 29,1 & 27 & 26,2 & 31 & 30,0 & 7 & 6,7 & 8 & 7,7 \\
\hline Masculino & 33 & 25,7 & 34 & 26,5 & 40 & 31,2 & 9 & 7,0 & 12 & 9,3 \\
\hline \multicolumn{11}{|c|}{ Clasificación del deporte } \\
\hline Combate & 19 & 34,5 & 11 & 20 & 15 & 27,2 & 3 & 5,4 & 7 & 12,7 \\
\hline Tiempo y marca & 24 & 31,1 & 23 & 29,8 & 18 & 23,3 & 8 & 10,3 & 4 & 5,1 \\
\hline Pelotas y raqueta & 19 & 19,5 & 27 & 27,8 & 37 & 38,1 & 5 & 5,1 & 9 & 9,2 \\
\hline Arte y precisión & 1 & 50 & - & - & 1 & 50 & - & - & - & - \\
\hline \multicolumn{11}{|c|}{ Clasificación de la lesión } \\
\hline Aguda & 1 & 1,1 & 8 & 9,3 & 58 & 67,4 & 7 & 8,1 & 12 & 13,9 \\
\hline Sobrecarga & 59 & 42,1 & 52 & 37,1 & 13 & 9,2 & 9 & 6,4 & 7 & 5 \\
\hline Sobrecarga y aguda & 2 & 100 & - & - & - & - & - & - & - & - \\
\hline Sobrecarga y & 1 & 100 & - & - & - & - & - & - & - & -- \\
\hline \multicolumn{11}{|c|}{ Zona Lesionada } \\
\hline $\begin{array}{l}\text { Miembros } \\
\text { superiores }\end{array}$ & 33 & 34 & 14 & 14,1 & 30 & 30,9 & 11 & 11,3 & 9 & 9,2 \\
\hline Tórax y tronco & - & - & 18 & 81,8 & - & - & - & - & 4 & 18,1 \\
\hline $\begin{array}{l}\text { Miembros } \\
\text { inferiores }\end{array}$ & 29 & 26,3 & 28 & 25,4 & 41 & 37,2 & 2 & 1,8 & 10 & 9,0 \\
\hline $\begin{array}{l}\text { Tronco y miembros } \\
\text { inferiores }\end{array}$ & 1 & 50 & 1 & 50 & - & - & - & - & - & - \\
\hline
\end{tabular}

\section{Discusión}

Este estudio, se convierte en el primer insumo epidemiológico para las ligas deportivas en el departamento del Cauca, donde el principal resultado de la investigación es la incidencia de las lesiones durante seis trimestres de entrenamiento y competencia, que pueden causar en el deportista disminución de la condición física, factores psicológicos adversos y bajo rendimiento, lo cual debe dar orientaciones a técnicos, entrenadores y preparadores físicos sobre las variantes e individualidades de cada deportista.

Estudios de Osorio et al.12, reportan que las tasas de lesiones de la práctica deportiva general oscilan entre 0,8 y 90,9 por 1000 horas de entrenamiento; este estudio reporta un promedio de aparición de lesión deportiva en general de $0,6 / 1000$ horas de entrenamiento, esta diferencia es entendible por la diversidad existente entre cada deporte, lastimosamente por la forma de clasificación de variables en este estudio, no permitió determinar la tasa de lesión deportiva diferencial para cada deporte.

Respecto a la edad de ocurrencia de las lesiones deportivas, este estudio agrupa una población con una mediana de 18 años, formado principalmente por estudiantes de colegios y universidades, El Centro de Control y Prevención de Enfermedades (CDC) de Atlanta reporta que, más de la mitad de los 7 millones de lesiones están relacionadas con actividades deportivas de alta competencia y actividades recreativas que ocurren anualmente se presentan en niños y jóvenes entre los 5 y 24 años de edad ${ }^{13}$, este estudio no individualiza 
cuantos lesionados se originan específicamente por la práctica deportiva de alta competencia, pero si hace una invitación a trabajar en la prevención de lesiones producto de una práctica deportiva.

Respecto a la incidencia de lesiones, el estudio reportó que las disciplinas deportivas con mayor frecuencia son: judo, futbol y voleibol. El judo, como deporte de contacto presenta un número importante de lesiones relacionadas con el impacto del deportista contra el suelo, los movimientos explosivos, giros, barridos $\mathrm{y}$ entradas o llaves del judoca, características de este deporte, observándose alta frecuencia de tendinopatía. Los resultados del estudio de García, manifiestan que casi 3 de cada 4 judocas han sufrido su lesión más importante en el transcurso de una competición ${ }^{14}$. Respecto al futbol, es un deporte con una alta incidencia de lesiones tanto en entrenamiento como en competición ${ }^{15}$. En lo referente al voleibol, debido a la rapidez y potencia de sus movimientos en sentido vertical y horizontal, la gran incidencia de lesiones es inevitable ${ }^{16}$.

En torno a las lesiones más frecuentes en deportistas, este estudio reporta las tendinopatías con mayor frecuencia en deportes de combate, de tiempo y marca; la lesión ligamentaria en los deportes de pelota y raqueta; mientras que la lesión muscular fue la más frecuente en los deportes de tiempo y marca; la zona de lesión de mayor compromiso para las tendinopatías fueron los miembros superiores, mientras que para las afecciones ligamentosas los miembros inferiores. El estudio de Leadbetter y Wayne ${ }^{13}$ reporta que un gran porcentaje de las lesiones sufridas durante la práctica del deporte, comprometen los tejidos blandos tales como: músculos, tendones, ligamentos y articulaciones, similar a lo encontrado en este estudio. Whitman et al., reportan que las áreas de mayor frecuencia de lesión en deportistas fueron: rodilla, tobillo y hombro, este estudio reporta como frecuentes la rodilla, el tobillo y el muslo 5 .

Son muchos los factores de riesgo que contribuyen a que se presente una lesión deportiva, este estudio por ser de tipo descriptivo no puede realizar afirmaciones sobre causalidad de la lesión, pero si puede recomendar para nuevas investigasiones determinar factores de riesgos y mecanismos de lesión, horas de entrenamiento, análisis biomecánico de la técnica deportiva, análisis postural, determinación de patrones de sueño y estrés para cada disciplina deportiva.

Los resultados de este estudio permitieron entregar a los directivos de Indeportes Cauca un programa de prevención de lesiones teniendo en cuenta la clasificación de la práctica deportiva, la clasificación de la lesión y la zona de mayor incidencia de lesión, enmarcando el programa de prevención en actividades de entrenamiento de la propiocepción de miembros inferiores, estabilidad del core, flexibilidad y fuerza excéntrica, basados en los principios del entrenamiento deportivo: individualización, especificidad, multilateralidad, continuidad e intensidad. Se propone que el programa se desarrolle con una frecuencia de tres veces por semana, con una duración de 30 minutos, y sea aplicado antes de iniciar la práctica deportiva habitual, este programa permitirá identificar por cada disciplina deportiva, las lesiones producidas por sobrecarga, sobreuso o sobre esfuerzo en la amplitud de movimiento articular y realizar los correctivos pertinentes en el corto plazo. El programa propuesto se basa en el modelo TRIPP que además de su aplicación requiere evaluar la eficacia de los protocolos de intervención además de valorar la relación riesgo-beneficio de la aplicación de la medida preventiva ${ }^{17}$, para determinar su efecto para cada disciplina deportiva.

\section{Conclusiones}

El presente estudio entrega un avance importante a los entes gubernamentales y a las ligas deportivas del Departamento del Cauca, a fin de documentar las lesiones por cada práctica deportiva con la finalidad de desarrollar un programa de prevención y seguimiento a las lesiones lo cual redundará en el rendimiento de las ligas y en los resultados de los deportistas Caucanos. 


\section{Agradecimientos}

Agradecemos a Indeportes Cauca, a los estudiantes de Fisioterapia de la Universidad del Cauca matriculados en el Enfasis en Deporte, por su colaboración y al Departamento de Fisioterapia de la Universidad del Cauca por su compromiso con la investigación.

\section{Conflicto de intereses: Ninguno declarado por} los autores.

\section{Referencias}

1. Bahr R, Maehlum S, Lesiones deportivas diagnóstico, tratamiento y rehabilitación. Ed Panamericana. 2007; 1: 3- 5.

2. Adamuz F, Nerin MA. El fisioterapeuta en la prevención de lesiones del deporte. Rev Fisioter. 2006; 5(2):31-36

3. Maffulli N. Types and epidemiology of tendinopathy. Clin Sports Med. 2003;22:675-692.

4. Pérez J. Las lesiones en el deporte Revista Cubana de Medicina del Deporte. 2010;5(1).

5. Moreno C. Epidemiología de las lesiones deportivas, Fisioterapia 2008;30(1):40-8

6. Junge A, Langevoort G, Pipe A, Peytavin A, Wong F, Mountjoy $\mathrm{M}$, et al. Injuries in team sport tournaments during the Olympic games. American Journal of Sports Medicine. 2004;(34):565-576.

7. Podlog L, Dimmock J, Miller J. A review of return to sport concerns following injury rehabilitation: Practitioner strategies for enhancing recovery outcomes. Physical Therapy in Sport. 2001;12(1):36-42.

8. Hagglund M, Walden M, Till L, Pruna R. Importancia de la epidemiologia en medicina del deporte. Apunts meds Esport.2010;45(166):57-59.

9. Ramos CA. Caracteristicas de las lesiones deportivas en el Taekwondo: Aspectos básicos de su tratamiento. Revista EDU-FISICA Grupo de Investigación Edufisica disponible en: http://www.edu-fisica.com Consultada Enero 15 de 2016.

10. Sanchez T, Castro L. Lesiones deportivas de las categorías mayores del club de patinaje Tequendama de Bogotá. Rev. Movimiento científico, 2015;9(1):21-32

11. The Helsinki Declaration Orvosi Hetilap 1965;106(3\&): 1715-1716.

12. Osorio C, Clavijo M, Arango E, Patiño S, Gallego Isabel. Lesiones deportivas. IATREIA, 2007;20(2):67-77

13. Leadbetter W, Wayne B. Soft tissue athletic injury. In: Stone D, Fu F, eds. Sports Injuries:mechanisms,prevention, and treatment. 2 ed. Philadelphia:Lippincott Williams \& Wilkins; 2001: 83988.

14. Garcia E. Lesiones en el judo de alta competición, actuación del deportista ante las mismas y valoración de los tratamientos de fisioterapia. Rev. Fisioterapia. 2008; 30(2):79-86.

15. Cos F. Modelos de análisis para la prevención de lesiones en el deporte. Estudio epidemiológico de lesiones: El modelo Union of European Football Associations en el fútbol, Apunts Med Esport. 2010;45(166):95-102.

16. Verhagen EA, van der Beek AJ, Bouter LM, Bahr RM, van Mechelen W. A one season prospective cohort study of volleyball injuries. Br J Sports Med. 2004; 38:477-81.

17. Finch C. A new framework for research leading to sports injury prevention. Journal of science and medicine in sport. 2006;9(1-2):3-9. Disponible en: http://www.sciencedirect.com/science/article/pii/S14 40244006000235 consultado el 6 de febrero de 2016 